\title{
Radiative hydrodynamic modeling of the Bastille-Day flare (14 July, 2000)
}

\section{Numerical simulations}

\author{
D. Tsiklauri ${ }^{1}$, M. J. Aschwanden ${ }^{2}$, V. M. Nakariakov ${ }^{3}$, and T. D. Arber ${ }^{3}$ \\ 1 Joule Physics Laboratory, School of Computing, Science \& Engineering, University of Salford, Salford M5 4WT, UK \\ 2 Lockheed Martin, Advanced Technology Center Solar \& Astrophysics Laboratory, Dept. L9-41, Bldg. 2523251 Hanover \\ Street, Palo Alto, CA 94304, USA \\ 3 Physics Department, University of Warwick, Coventry CV4 7AL, UK
}

Received 23 January 2004 / Accepted 26 February 2004

\begin{abstract}
A 1D loop radiative hydrodynamic model that incorporates the effects of gravitational stratification, heat conduction, radiative losses, external heat input, presence of helium, and Braginskii viscosity is used to simulate elementary flare loops. The physical parameters for the input are taken from observations of the Bastille-Day flare of 2000 July 14. The present analysis shows that: a) the obtained maximum values of the electron density can be considerably higher $\left(4.2 \times 10^{11} \mathrm{~cm}^{-3}\right.$ or more $)$ in the case of footpoint heating than in the case of apex heating $\left(2.5 \times 10^{11} \mathrm{~cm}^{-3}\right)$; b) the average cooling time after the flare peak takes less time in the case of footpoint heating than in the case of apex heating; c) the peak apex temperatures are significantly lower (by about $10 \mathrm{MK}$ ) for the case of footpoint heating than for apex heating (for the same average loop temperature of about $30 \mathrm{MK}$ ). This characteristic would allow to discriminate between different heating positioning; d) in both cases (of apex and footpoint heating), the maximum obtained apex temperature $T^{\max }$ is practically independent of the heating duration $\sigma_{t}$, but scales directly with the heating rate $\left.E_{\mathrm{H} 0} ; \mathrm{e}\right)$ the maximum obtained densities at the loop apex, $n_{\mathrm{e}}^{\max }$, increase with the heating rate $E_{\mathrm{H} 0}$ and heating duration $\sigma_{t}$ for both footpoint and apex heating. In Paper II we will use the outputs of these hydrodynamic simulations, which cover a wide range of the parameter space of heating rates and durations, as an input for forward-fitting of the multi-loop arcade of the Bastille-day flare.
\end{abstract}

Key words. Sun: flares - Sun: activity - Sun: corona

\section{Introduction}

Solar flares are complex systems that involve many magnetic field lines and thus can rarely be represented by a single fluxtube. During the Bastille-Day 2000 July 14 flare for instance, which exhibits a classical double-ribbon flare configuration, an ensemble of over 200 individual post-flare loops has been identified (Aschwanden \& Alexander 2001). Each of these individual loops has its own hydrodynamic evolution during a flare, occurring in magnetic flux systems that are thermodynamically isolated from each other and have their own independent timing and physical parameters. Hydrodynamic modeling of flare loops, however, have been performed for single flare loops (Mariska 1987; Mariska et al. 1989), but only few MHD simulation studies have been orchestrated in a multi-loop configuration (Hori et al. 1997, 1998). Even the multi-loop simulations have been designed only in the simplest way, by assuming regular spacing and time intervals to merely mimic the

Send offprint requests to: $\mathrm{D}$. Tsiklauri,

e-mail: D.Tsiklauri@salford.ac.uk superposition effect, but no detailed fitting of the observed spatial configurations and timing has ever been attempted. A rigorous hydrodynamic modeling effort for complex large flares would be extremely valuable to constrain the total energy budget, the heating functions, the fractal geometric structure, the plasma filling factors, and the spatio-temporal organization of unsteady (Priest \& Forbes 2000) or impulsive bursty magnetic reconnection processes (Leboef et al. 1982), which are likely to occur in large double-ribbon flares because of the large shear and resulting tearing mode instability (Sturrock 1966).

In this series of papers we present a method of radiative hydrodynamic modeling of large, complex, multi-loop flares. In this Paper I we perform numerical simulations with a 1D hydrodynamic code to obtain the temperature evolution $T_{\mathrm{e}}^{\max }(t), T_{\mathrm{e}}^{\text {avg }}(t)$ and density evolution $n_{\mathrm{e}}^{\max }(t)$ in a large parameter space of heating functions. We vary the maximum heating rate, heating duration, and location (footpoint, apex) of the heating functions $E_{\mathrm{H}}(t)$. In Paper II we parameterize the results, suitable to forward-fitting of a multi-loop system and fit the multi-wavelength data of the Bastille-day flare 2000 July 14 
using TRACE, Yohkoh/SXT, HXT, and GOES data. In subsequent papers we plan to extend the hydrodynamic results of this flare to constrain magnetic modeling, magnetic reconnection geometries, and particle acceleration processes.

\section{The radiative hydrodynamic model}

In order to describe plasma dynamics in a coronal loop, we solve numerically $1 \mathrm{D}$ radiative hydrodynamic equations (infinite magnetic field approximation) that resemble closely the Naval Research Laboratory Solar Flux Tube Model (SOLFTM) (Mariska 1987). The numerical code that we use is a $1 \mathrm{D}$ version of the Lagrangian Re-map code (Arber et al. 2001) with radiative loss limiters. As in SOLFTM the coronal loop is connected with a dense, cold $(10000 \mathrm{~K}), 5 \mathrm{Mm}$ thick plasma region, that mimics the chromosphere, and which because of its large density provides sufficient amount of matter to fill the loop during the flare. The use term of "chromosphere" should be taken with caution, as we do not consider realistic one (with ionizations, proper radiative transfer, etc.). Instead, we simply mimic chromosphere by considering it as a dense, cold (10000 K) plasma, which provides source of matter inflow into the loop during the flare, while we use radiative loss limiters to prevent plasma from catastrophic cooling, as we use optically thin plasma approximation $\left(\propto n_{\mathrm{e}}^{2}\right)$. In such approach, specific initial conditions in region connecting corona to the chromosphere and chromosphere itself have little or no effect on the corona dynamics as they are rapidly modified, selfconsistently, according to the radiative hydrodynamics equations. In brief, our model includes: the effects of gravitational stratification, heat conduction, radiative losses, added external heat input, presence of helium, hydrodynamic non-linearity, and Braginskii bulk viscosity (Braginskii 1965). For the radiative loss function we use the following parameterization,

$$
L_{\mathrm{r}}(T)=n_{\mathrm{e}}^{2}\left\{\begin{array}{cc}
10^{-26.60} T^{1 / 2} & T>10^{7.6} \\
10^{-17.73} T^{-2 / 3} & 10^{6.3}<T<10^{7.6} \\
10^{-21.94} & 10^{5.8}<T<10^{6.3} \\
10^{-10.40} T^{-2} & 10^{5.4}<T<10^{5.8} \\
10^{-21.2} & 10^{4.9}<T<10^{5.4} \\
10^{-31} T^{2} & 10^{4.6}<T<10^{4.9} \\
10^{-21.85} & 10^{4.3}<T<10^{4.6} \\
10^{-48.31} T^{6.15} & 10^{3.9}<T<10^{4.3} \\
10^{-69.90} T^{11.7} & 10^{3.6}<T<10^{3.9}
\end{array}\right.
$$

which is an extension of Rosner et al. (1978) compiled from other sources (Priest 1982; Peres et al. 1982).

\section{Numerical results}

We start numerical simulations from the following configuration: we take a semicircular loop with a length of $L=$ $55 \mathrm{Mm}$ (which corresponds to an average loop arcade radius of $r=17.5 \mathrm{Mm}$, as derived by Aschwanden \& Alexander (2001), Table III, for the Bastille-day flare). We keep the coronal part of the loop initially at a temperature of $1 \mathrm{MK}$ and at a mass density of $\rho=\mu m_{\mathrm{p}} n_{\mathrm{e}}=6.6 \times 10^{-16} \mathrm{~g} \mathrm{~cm}^{-3}$ (at the loop apex), for a helium-to-hydrogen number density ratio of 0.05 , i.e., with a mean molecular weight of $\mu=(1+0.05 \times 4) /(1+0.05 \times 2)=1.1$, this corresponds to an electron density of $n_{\mathrm{e}}=3.6 \times 10^{8} \mathrm{~cm}^{-3}$. The resolution in all our numerical runs was fixed to 1000 grid points, which were distributed non-uniformly in order to properly resolve strong gradients in the region connecting corona to the chromosphere. As a convergence test, runs with 3000 grid points were made, which showed no difference to the case with 1000 grid points, thus confirming sufficient numerical resolution in our simulations.

\subsection{Heating function}

The heating function in flare loops is probably quite different from that of non-flaring loops. Flare loops are filled by upflowing heated plasma from the chromosphere, once the chromospheric footpoints become impulsively heated from precipitating non-thermal particles and/or downward propagating hot thermal conduction fronts from the coronal reconnection site. Chromospheric evaporation seems to be the main matter inflow source for flare loops. The heating function has therefore to accommodate very localized heating at the footpoints (Tsiklauri \& Nakariakov 2001; Aschwanden et al. 2001). On the other side, reconnection outflows from the reconnection region contain also heated plasma and heat the flare loops from the apex side, for instance in the standard reconnection model of Kopp \& Pneuman (1976), which seems to fit the magnetic configuration of the Bastille-day flare to first order (Aschwanden \& Alexander 2001). Thus, to allow for both options, we have performed numerical simulations for both cases separately, i.e., for apex and footpoint heating functions.

We have used the following heating function in our simulations:

$$
\begin{aligned}
E_{\mathrm{H}}(s, t) & =E_{\mathrm{H}}^{\mathrm{s}}(s) E_{\mathrm{H}}^{\mathrm{t}}(t)=E_{0}\left[\exp \left(-\frac{\left(s+s_{0}\right)^{2}}{2 \sigma_{\mathrm{s}}^{2}}\right)\right. \\
& \left.+\exp \left(-\frac{\left(s-s_{0}\right)^{2}}{2 \sigma_{\mathrm{s}}^{2}}\right)\right] \times\left[1+\alpha Q_{\mathrm{p}} \exp \left(-\frac{\left(t-t_{\mathrm{p}}\right)^{2}}{2 \sigma_{\mathrm{t}}^{2}}\right)\right] .
\end{aligned}
$$

Here, $E_{\mathrm{H}}^{\mathrm{s}}(s)$ and $E_{\mathrm{H}}^{\mathrm{t}}(t)$ are the spatial and temporal parts of the heating function, which are taken to be independent of each other for simplicity. $E_{0}$ is the heating rate in units of $\left[\mathrm{erg} \mathrm{cm}^{-3} \mathrm{~s}^{-1}\right]$. The positions $s= \pm s_{0}$ are the locations with the maximum heat deposition, i.e., $s_{0}=0$ for apex heating. The heat deposition length scale is called $\sigma_{\mathrm{s}}$ (i.e., the spatial width of the Gaussian). The temporal part of the heating function is similar to one used by Aschwanden \& Alexander (2001) (cf. their Eq. (31)), where $t_{\mathrm{p}}$ is the flare peak time and $\sigma_{\mathrm{t}}$ is the duration of the flare (i.e., the temporal width of the Gaussian). However, our choice of the temporal part of the heating function is such that there is a small background heating present at all times (either at footpoints or the loop apex) which ensures that in the absence of flare heating (when $\alpha=0$, a parameter that determines the flare heating amplitude), the average loop temperature stays at $1 \mathrm{MK}$. We defined the average loop temperature as the sum of temperatures along the loop divided by the number of grid points at a given snapshot.

For easy comparison between apex and footpoint heating cases we fix the flare heating amplitude $Q_{\mathrm{p}}$ at a given different 
value in each case. This ensures that the average loop temperature peaks at about at the observed value of $30 \mathrm{MK}$ in both cases, when the the flare heating is on $(\alpha=1)$. Then we vary also $\alpha=0.01,0.05,0.25,0.5,1.0$ to obtain different apex temperatures $T^{\max }$. Hereafter, we denote the (flare) heating rate by $E_{\mathrm{H} 0}=E_{0} \alpha Q_{\mathrm{p}}$.

In all our numerical runs presented here, $1 /\left(2 \sigma_{\mathrm{s}}^{2}\right)$ was fixed to a value of $0.01 \mathrm{Mm}^{-2}$, which gives a heat deposition length scale of $\sigma_{\mathrm{s}}=7 \mathrm{Mm}$. This is a typical value determined from observations (Aschwanden et al. 2002). The flare peak time was fixed in our numerical simulations at $t_{\mathrm{p}}=822 \mathrm{~s}$. The time step of data visualization (which in fact is much larger that the actual time step, $0.035 \mathrm{~s}$, in the numerical code) was chosen to be $\Delta t=10 \mathrm{~s}$.

We would like to close this subsection with following remarks about the heating function: first, in the case of the chromospheric footpoint heating one usually assumes that the source of heating is the non-thermal particles propagating downwards from the acceleration region (presumably located in the apex of the loop). Such type of heating source, however, could be efficient only in a cold $(T<10000 \mathrm{~K})$ and dense $\left(n_{\mathrm{e}}>10^{11} \mathrm{~cm}^{-3}\right)$ plasma, as compared to one considered in this model. The problem is that the mean free path of, e.g. $20 \mathrm{KeV}$ electrons in plasma with $T=10000 \mathrm{~K}$ and $n_{\mathrm{e}}=10^{11} \mathrm{~cm}^{-3}$ is about $27 \mathrm{Mm}$, which is much greater than the thickness of a real, as well as the model $(5 \mathrm{Mm})$, chromosphere. Thus, the collisional energy deposition from the nonthermal electrons possibly can not be efficient here. On the other hand the observations (Aschwanden et al. 2002) support claim that $\sigma_{\mathrm{s}} \approx 10 \mathrm{Mm}$ (we actually fixed it at $7 \mathrm{Mm}$ ). Thus, there a room for controversy in this issue. Besides, there can be sources of energy deposition other than the collisional one from the non-thermal electrons, such as reconnection occurring due to random motion of footpoints, etc. Also, often based solely on the "mean free path considerations" the entire continuous description (MHD or hydrodynamic) of plasma can be questionable, while there is still an evidence that these descriptions effectively work in spite of this. Second, if top of the loop is heated by the reconnection, then ideally the numerical model should include a source the injection of hot plasma into the apex region of the loop instead of just using spatially distributed impulsive heating. In the case of hot plasma injection from outside, the behaviour of the model will be certainly different from one presented in this paper. However, for the sake of simplicity, this effect is ignored here.

\subsection{Case of apex heating}

In the case of apex heating we fix $s_{0}=0 \mathrm{Mm}$ in Eq. (1). Initially we run our numerical code without flare heating, i.e. we put $\alpha=0$ (in this manner we turn off flare heating). In all our numerical runs we keep background heating on at all times, so that flare occurs starting from a steady, equilibrium loop. The result of this simulation is presented in Fig. 1. Dash-dotted curves in panels a) and b) show the evolution of the average and apex temperatures in time. Since the observational data of the Bastille day flare, namely the temperature evolution (cf. top left panel of Fig. 11 in Aschwanden \& Alexander 2001), was derived for the entire field of view of the instrument, we found that it is useful, in addition to the apex temperature, to track also the average loop temperature. Since the outputs from our hydrodynamic simulations will be used to model the observational data of the Bastille-day flare in Paper II, $E_{0}$ was fixed to $0.002 \mathrm{erg} \mathrm{cm}^{-3} \mathrm{~s}^{-1}$ in all runs of this subsection, which insures that the average loop temperature stays at $1 \mathrm{MK}$ in the absence of flare heating (cf. Fig. 1a). Note that the apex temperature in this case tends to have a higher asymptotic value of $2.5 \mathrm{MK}$ (cf. Fig. 1b). The obtained asymptotic value of the loop apex number density is $n_{\mathrm{e}}=0.9 \times 10^{9} \mathrm{~cm}^{-3}$ in this case (and thus is not visible in Fig. 1c).

Then, we run our numerical code with flare heating, i.e. we put $\alpha=1$ and fix $Q_{\mathrm{p}}=3 \times 10^{4}$, so that it yields peak average temperature of about $30 \mathrm{MK}$, as observed during the Bastilleday flare (Aschwanden \& Alexander 2001), and then we vary the duration of the heating phase to $\sigma_{\mathrm{t}}=41,164,329 \mathrm{~s}$ (i.e., the temporal width of the Gaussian). Note that it is the combined action of the heating rate $Q_{\mathrm{p}}$, the flare heating amplitude $\alpha$, and the duration $\sigma_{\mathrm{t}}$ of the flare, that determine the effect of flare heating, i.e. the term $\alpha Q_{\mathrm{p}} \exp \left(-\left(t-t_{\mathrm{p}}\right)^{2} / 2 \sigma_{\mathrm{t}}^{2}\right)$ in Eq. (1), compared with the background heating rate $(\alpha=0)$. In fact, some of the considered values of $\sigma_{\mathrm{t}}$, e.g. $329 \mathrm{~s}$, imply that right at the very beginning initial equilibrium loop (resting at about $1 \mathrm{MK}$ ) is subjected to a substantial increase of heat input. In this manner, we are trying model some of most violent flare events. The results are presented in Fig. 1. There are several noteworthy features in this graph: (1) as expected, an increase of the heating time interval $\sigma_{\mathrm{t}}$ yields an increase of the flare duration (cf. Figs. 1a,b); (2) however, an increase in the duration of the heating phase does not affect the obtained maximum values of the average nor the apex temperature (cf. Figs. 1a and 1b). This is counter-intuitive, because we expect that the amount of deposited heat increases with the heating duration. This invariance of the obtained flare temperatures with respect to duration of the flare could probably be explained by some form of saturation (or balance) in the combined action of losses in the system - heat conduction and radiative losses - and flare heat input; (3) an increase in the duration of heating should naturally result in an increase of the plasma density in the loop. For the long duration flares (the thickest solid line in Fig. 1c) there are three clearly different physical regimes which yet have to be properly identified; (4) very useful information can be extracted from the velocity outputs at the footpoints $(s= \pm 27.5)$ and at the apex $(s=0)$, as function of time (cf. Figs. 1d-f). We find that strong up-flows (up to $400 \mathrm{~km} \mathrm{~s}^{-1}$ ) are present at the footpoints of the flare loops during the flare onset, as the deposited heat (delivered by conduction from the apex to the footpoints) causes material evaporation from the dense chromosphere. The late up-flow phase is followed by an oscillatory phase with typical amplitudes of a few tens of $\mathrm{km} \mathrm{s}^{-1}$, which in turn is followed by down-flows (up to $100 \mathrm{~km} \mathrm{~s}^{-1}$ ), when plasma is drained out of the loop; (5) note that the velocity dynamics at opposite footpoints remains perfectly symmetric at all times (cf. Figs. 1d-f), while net flow through the apex remains zero at all times as expected. This is due to the symmetry of the problem; (6) the peak up-flow velocity during the 

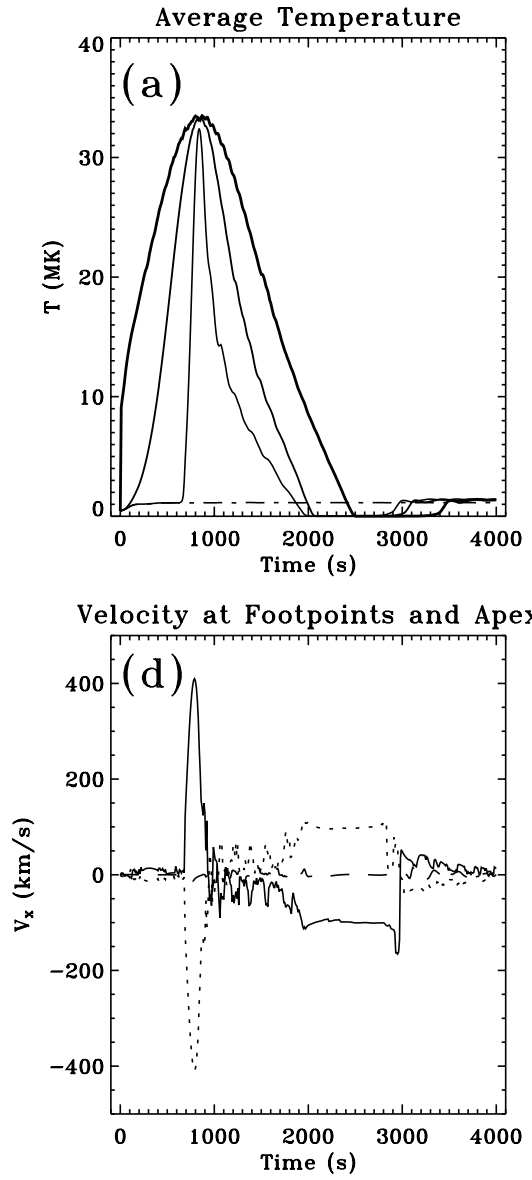

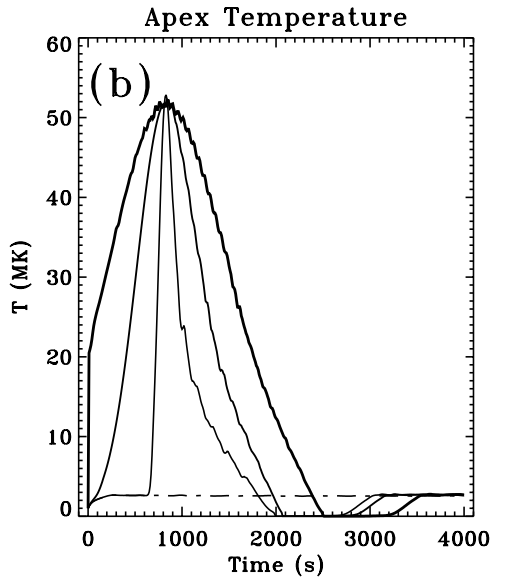

Velocity at Footpoints and Apex

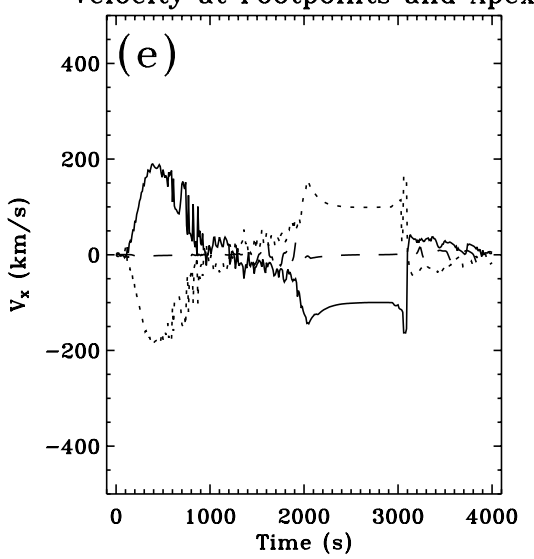

Loop Apex N. Density
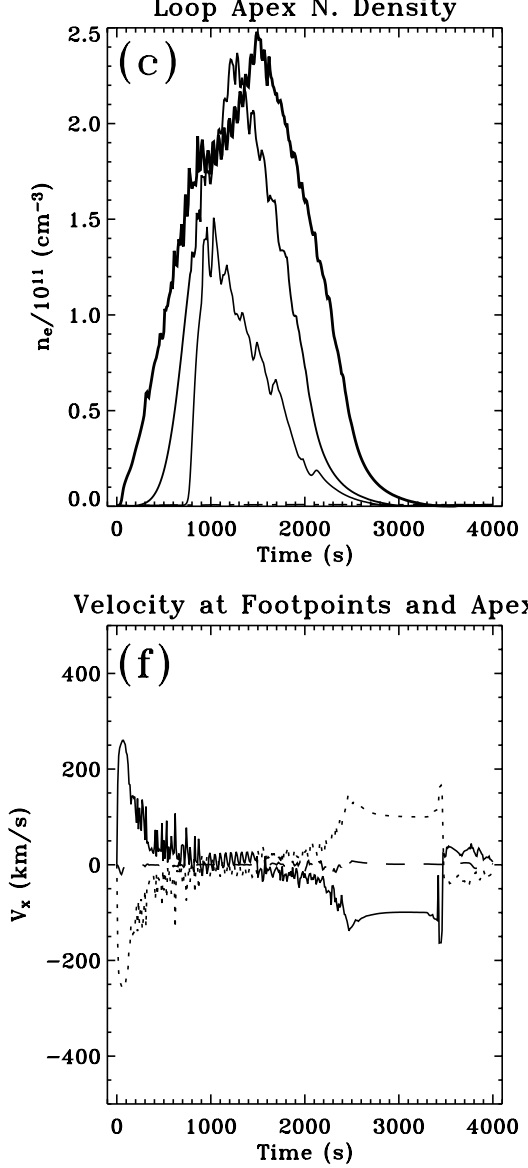

Fig. 1. a) Average temperature as a function of time in the case of apex heating. Dash-dotted curve corresponds to the case of background heating $(\alpha=0)$. The thin, thick and thickest solid lines correspond to the cases of background plus flare heating $(\alpha=1)$ for different flare durations, $\sigma_{\mathrm{t}}=41,164,329 \mathrm{~s}$, respectively. b) The same as in a), but for the temperature at the apex. c) The same as in a), but for the number density at the apex. d) Velocities versus time at footpoints $(s= \pm 27.5)$, solid and dotted curves, and apex $(s=0)$, dashed curve, for the case of background plus flare heating ( $\alpha=1$ ) for the flare duration, $\sigma_{\mathrm{t}}=41 \mathrm{~s}$. e) The same as in d), but for $\sigma_{\mathrm{t}}=164 \mathrm{~s}$. f) The same as in d), but for $\sigma_{\mathrm{t}}=329 \mathrm{~s}$.

onset of a flare decreases with the increase of the duration of the flare (for $\sigma_{\mathrm{t}}=41 \mathrm{~s}$ ). In Fig. 1d the peak up-flow velocity is about $400 \mathrm{~km} \mathrm{~s}^{-1}$, while for $\sigma_{\mathrm{t}}=164 \mathrm{~s}$ (Fig. 1e), it is about $200 \mathrm{~km} \mathrm{~s}^{-1}$ ). This may seem counter-intuitive at first glance, since we would expect that the duration of the up-flow itself increases with increasing heating duration $\sigma_{\mathrm{t}}$. However, the net material evaporated into the loop from chromosphere still increases with the increase of the heating duration, so that the densities obtained at the apex increase (cf. Fig. 1c); (7) yet another interesting observation is about the time instances when down-flows abruptly end after the flare (cf. Figs. 1d-f), these times correspond exactly to the same time instances when both the average and apex temperature curves reach these asymptotic values (thin, thick, and very thick solid lines that join the dash-dotted lines in Figs. 1a and 1b), signaling the end of the flare heating phase and the onset of a steady state. In fact, statements (6) and (7) are true for all numerical runs performed (see below).

In a next step we investigate the effect of the flare heating amplitude, by fixing the duration of the flare $\sigma_{\mathrm{t}}=41 \mathrm{~s}$ and varying $\alpha=0.01,0.25,1.0$ (again with fixed $Q_{\mathrm{p}}=3 \times 10^{4}$ ). The results of these numerical runs are presented in Fig. 2.
There are several interesting features in this graph: (1) as expected, a decrease of $\alpha$ results in a decrease of the obtained flare temperature (cf. Figs. 2a and 2b); (2) also, a decrease of the flare heating amplitude does not affect the duration of the flare (cf. Figs. 2a and 2b); (3) in addition, a decrease of the flare heating amplitude naturally results in a decrease of the plasma density in the loop. This is understandable as the less deposited heating rate causes less material evaporation form the chromosphere and, hence, produces less dense and cooler loops during the flare (cf. Figs. 2a-c); (4) again, as in Figs. 1d-f, we studied the resulting velocities at the footpoints $(s= \pm 27.5 \mathrm{Mm})$ and apex $(s=0)$ as function of time (cf. Figs. $2 d-f$ ). Our simulations show that the peak up-flow velocities during the onset of the flare decrease with decreasing heating amplitudes. For $\alpha=1.0$ (Fig. 1d), the peak up-flow velocity is about $400 \mathrm{~km} \mathrm{~s}^{-1}$, while for $\alpha=0.01$ (Fig. 1f), it is about $160 \mathrm{~km} \mathrm{~s}^{-1}$. Also, note that the 4-fold decrease in the heating flare amplitude (Fig. 1e) still results in down-flows of the order of $100 \mathrm{~km} \mathrm{~s}^{-1}$, which are typical for most runs, while further decrease in $\alpha$ (Fig. 1d) causes an absence of any noticeable down-flows. 

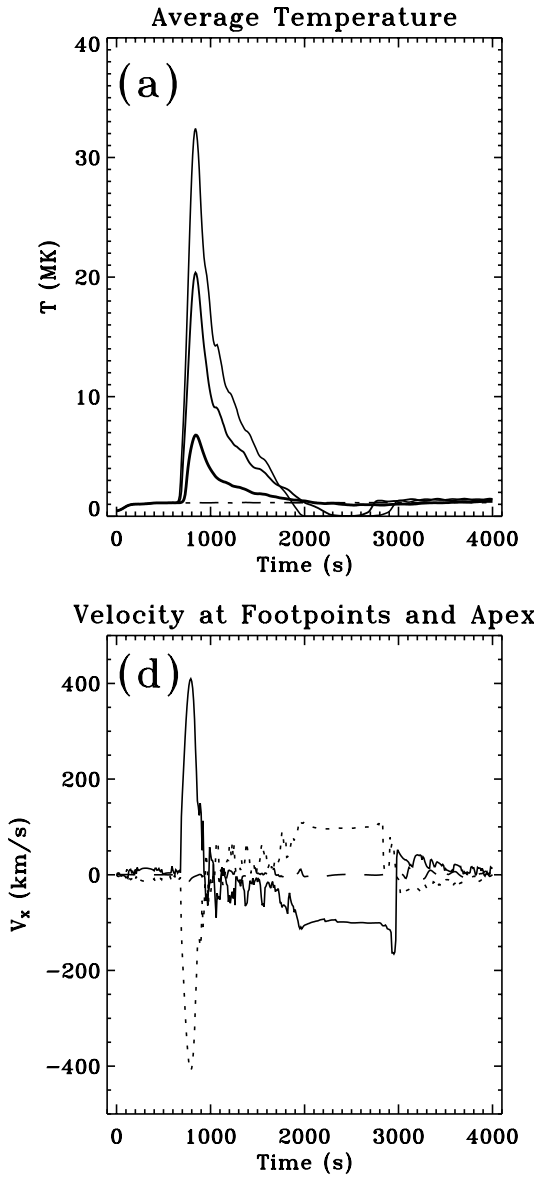
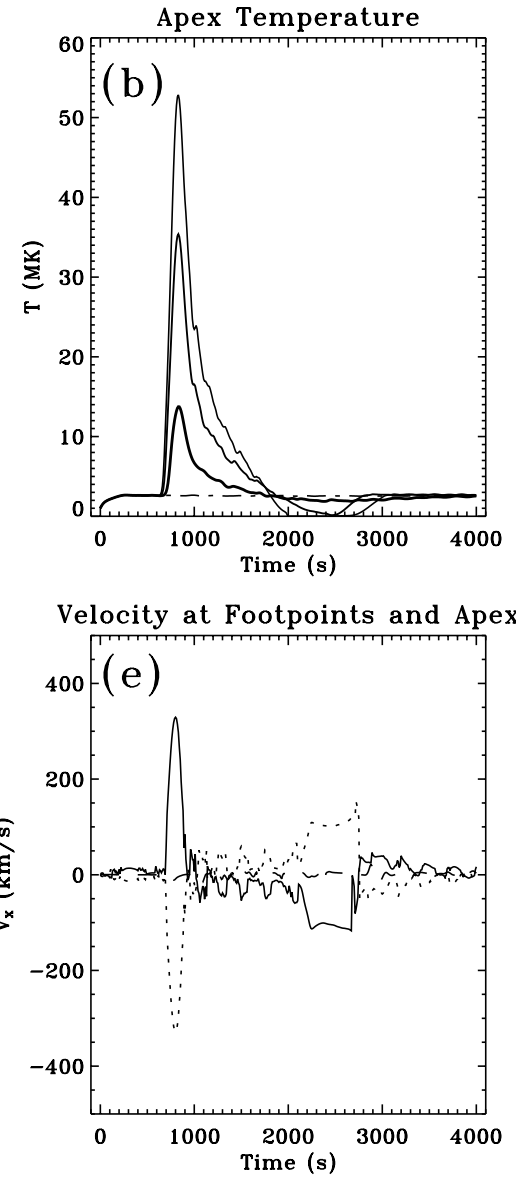
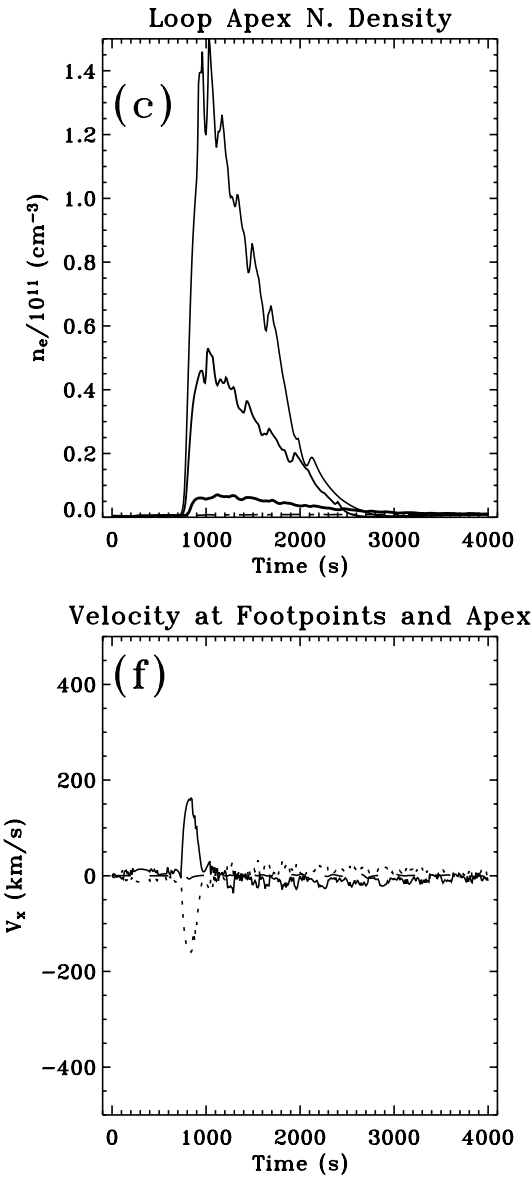

Fig. 2. a) Average temperature as a function of time in the case of apex heating. Dash-dotted curve corresponds to the case of background heating $(\alpha=0)$. The thin, thick and thickest solid lines correspond to the cases of background plus flare heating for different flare peak amplitudes, $\alpha=0.01,0.25,1.0$, respectively (these correspond to flare heating rates $E_{\mathrm{H} 0}=0.6,15.0,60.0 \mathrm{erg} \mathrm{cm}^{-3} \mathrm{~s}^{-1}$ ). b) The same as in a), but for the temperature at the apex. c) The same as in a), but for the number density at the apex. d) Velocities versus time at footpoints ( $s= \pm 27.5$ ), solid and dotted curves, and apex $(s=0)$, dashed curve, for the case of background plus flare heating (flare duration $\sigma_{\mathrm{t}}=41 \mathrm{~s}$ ) for the flare peak amplitude, $\alpha=1.0$ (which corresponds to flare heating rate $E_{\mathrm{H} 0}=60.0 \mathrm{erg} \mathrm{cm}^{-3} \mathrm{~s}^{-1}$ ). e) The same as in d), but for $\alpha=0.25$ $\left(E_{\mathrm{H} 0}=15.0 \mathrm{erg} \mathrm{cm}^{-3} \mathrm{~s}^{-1}\right)$. f) The same as in d), but for $\alpha=0.01\left(E_{\mathrm{H} 0}=0.6 \mathrm{erg} \mathrm{cm}^{-3} \mathrm{~s}^{-1}\right)$.

\subsection{Case of footpoint heating}

In the case of footpoint heating we fix $s_{0}= \pm 30 \mathrm{Mm}$ in Eq. (1), i.e. the (spatial) peaks of the heating are chosen to be at the bottom of the region connecting corona to the chromosphere (i.e. top of chromosphere). As for the case of apex heating, we run initially our numeric code without flare heating $(\alpha=0)$. The output from this numerical run of the code is presented in Fig. 3. $E_{0}$ is in all runs in this subsection fixed to a value of $0.01 \mathrm{erg} \mathrm{cm}^{-3} \mathrm{~s}^{-1}$, which insures that the average loop temperature stays at $1 \mathrm{MK}$ in the absence of flare heating (cf. Fig. 3a). Note that in the case footpoint heating, the amount of deposited heat required to keep the loop at an average temperature of $1 \mathrm{MK}$ is 5 times greater than in the case of apex heating (recall that in the later case $E_{0}=0.002 \mathrm{erg} \mathrm{cm}^{-3} \mathrm{~s}^{-1}$ ). This increased heating is required in order: (A) to compensate the increased radiative losses (that are $\propto n_{\mathrm{e}}^{2}$ ) in the relatively dense chromosphere and low corona and (B) for the upward heat conduction to be sufficient enough to keep the loop at this temperature, with the latter point (B) being probably less important than the former one (A). The apex temperature in the case of footpoint heating approaches an asymptotic value of 1.7 MK (cf. Fig. 3a), while the obtained asymptotic value of the loop apex number density is $0.02 \times 10^{11} \mathrm{~cm}^{-3}$ in this case (not visible in Fig. 3c). Note that the asymptotic value of the loop apex temperature, $T^{\mathrm{max}}=1.7 \mathrm{MK}$, is lower in the case of footpoint heating (due to the flatness of the temperature spatial profile along the loop) compared with the case of apex heating $\left(T^{\max }=2.5 \mathrm{MK}\right)$. The larger amount of deposited heat in the case of footpoint heating results in an asymptotic value of the the loop apex density $\left(n_{\mathrm{e}}=0.02 \times 10^{11} \mathrm{~cm}^{-3}\right)$ that is more than twice the value for apex heating $\left(n_{\mathrm{e}}=0.009 \times 10^{11} \mathrm{~cm}^{-3}\right)$. This is due to the fact that the more deposited heat causes more material evaporation from the chromosphere into the loop.

Yet another interesting point that only appears in the case of footpoint heating is that there is a minimal heat deposition length scale, $\sigma_{\mathrm{s}}$, which allows the existence of a steady loop at an average temperature of $1 \mathrm{MK}$. The results presented in this subsection are for $\sigma_{\mathrm{s}}=7 \mathrm{Mm}$. Smaller values cause a gradual formation of a condensation at the apex of the loop (clearly seen in the animations, not included here), which then forms a prominence due to the thermal instability and causes 

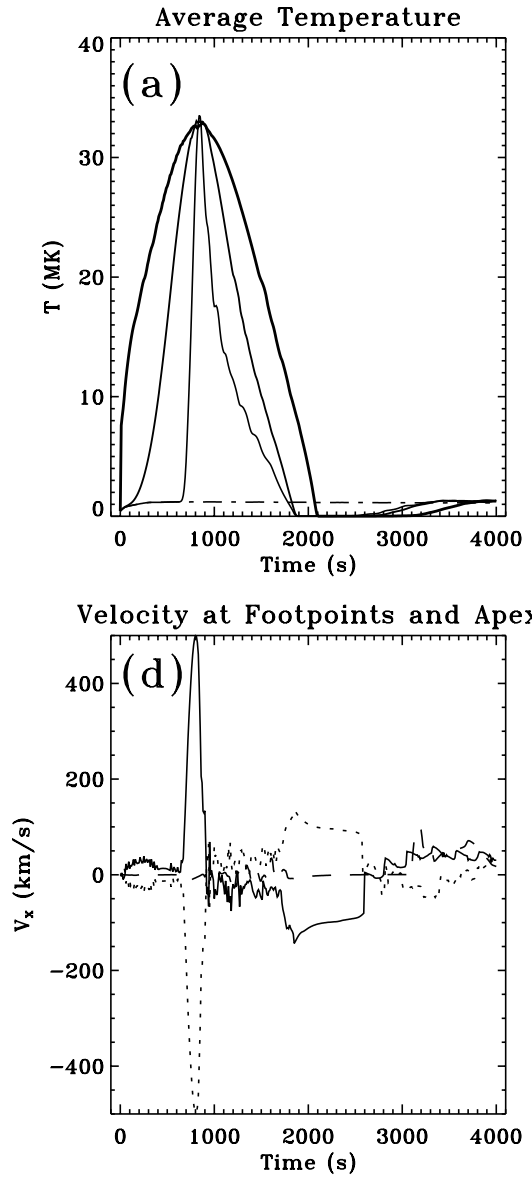

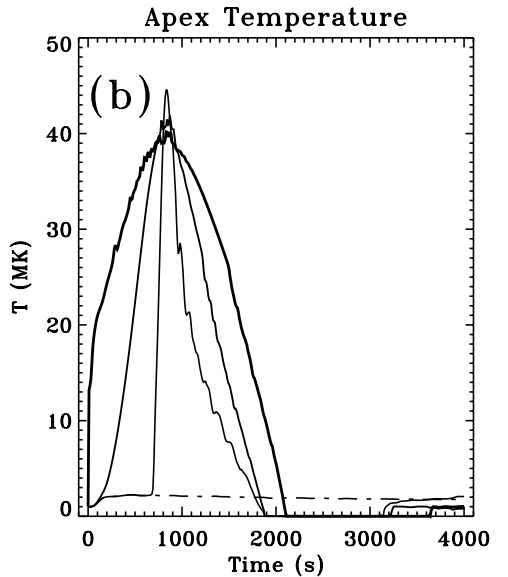

Velocity at Footpoints and Apex

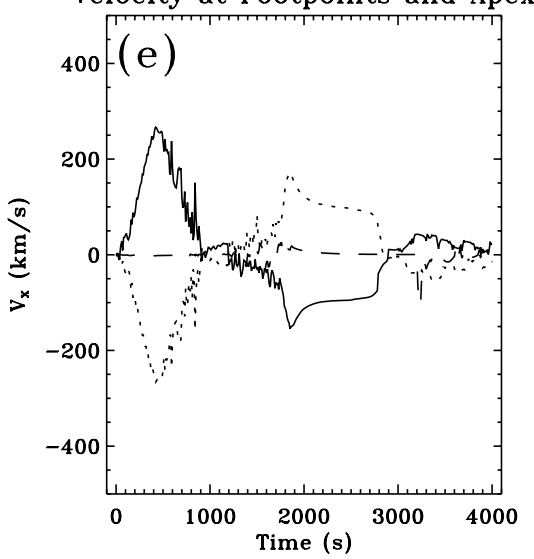

Loop Apex N. Density

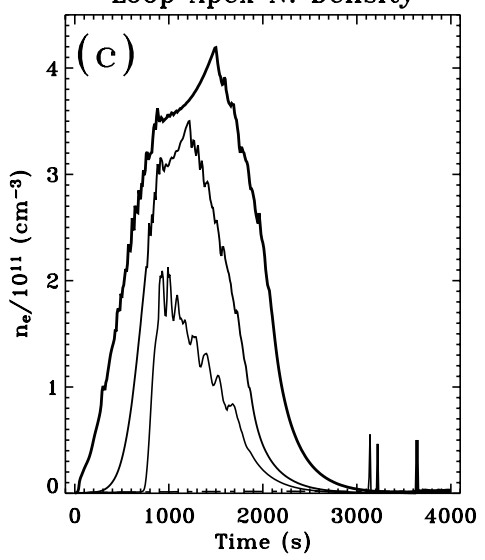

Velocity at Footpoints and Apex

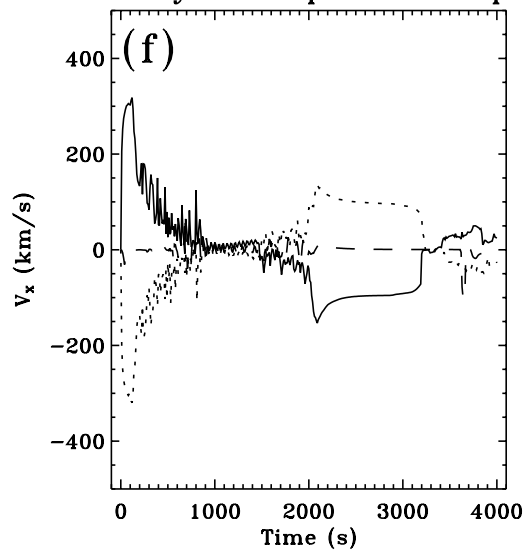

Fig. 3. The same as in Fig. 1, but for footpoint heating.

an eventual disappearance of the loop. This occurs even with a substantial increase of the heating amplitude $E_{0}$. This is caused by the inefficiency of upward heat conduction to keep the loop at a typical coronal temperature of the order of $T \approx 1 \mathrm{MK}$.

Our next step is to switch on flare heating as described in previous subsection. The physical parameters used here are also the same (apart from differences in the steady state as described above) for easy comparison. Namely, we put $\alpha=1$, and fix $Q_{\mathrm{p}}=1.5 \times 10^{4}$, so that it yields an average peak temperature of about $T^{\max }=30 \mathrm{MK}$ as in the Bastille-day flare (Aschwanden \& Alexander 2001), and we vary duration of the flare by changing the heating time scale to $\sigma_{\mathrm{t}}=41,164,329 \mathrm{~s}$. The results of this simulation are presented in Fig. 3. Since, the dynamics of flare with variation of its duration has been described in considerable detail for the case of apex heating (Fig. 1), we focus now our attention on differences between the two cases. These can be summarized as follows: (1) we observe (Fig. $3 \mathrm{c}$ ) that the obtained maximum values of the density are considerably higher $\left(n_{\mathrm{e}}=4.2 \times 10^{11} \mathrm{~cm}^{-3}\right)$ in the case of footpoint heating than in the case of apex heating $\left(n_{\mathrm{e}}=2.5 \times\right.$ $10^{11} \mathrm{~cm}^{-3}$ ), (compare Fig. 3c with Fig. 1c). This is due to the fact that footpoint heating is more efficient in evaporating material from the region connecting corona to the chromosphere and chromosphere itself, yielding denser loops during the flare. In fact, previous hydrodynamic simulations assert that there is a maximum density limit, no matter how much heat is deposited.
This limiting density is about $2 \times 10^{11} \mathrm{~cm}^{-3}$ (Mariska et al. 1989, cf. their Fig. 2, after 60 s). Fisher \& Hawley (1990) get similar values. An analogous problem exists in quite Sun loops. Aschwanden et al. (2001) points out that the observed TRACE loops show a higher density and pressure than expected from the RTV law, which we might call the "over-density loop problem". However, as we can see in Fig. 3c, it is possible to break that "density limit" and the reason for this is that we have used footpoint heating. In the case of footpoint heating it is possible to evaporate more material from the chromosphere and the region connecting corona to the chromosphere, where the density is orders of magnitude higher than in the corona. In the case of apex heating, in contrast, insufficient downward heat conduction prevents significant evaporation. Works of Mariska et al. (1989) and Fisher \& Hawley (1990) presumably were motivated to use apex heating because of the standard reconnection model (Kopp \& Pneuman 1976), which implies that the heating function is localized in the loop apex (actually, Mariska et al. (1989) used a model in which the (collisional) heating by a beam of non-thermal electrons injected at the apex followed the density evolution of the flare loop and so varied in time in response to the evolution of the flare). Consequently they obtain lower densities in the loop. Similar results were obtained by Warren et al. (2003). They showed that by modeling a loop as set of small scale, impulsively heated filaments one can generally reproduce the observed dense 

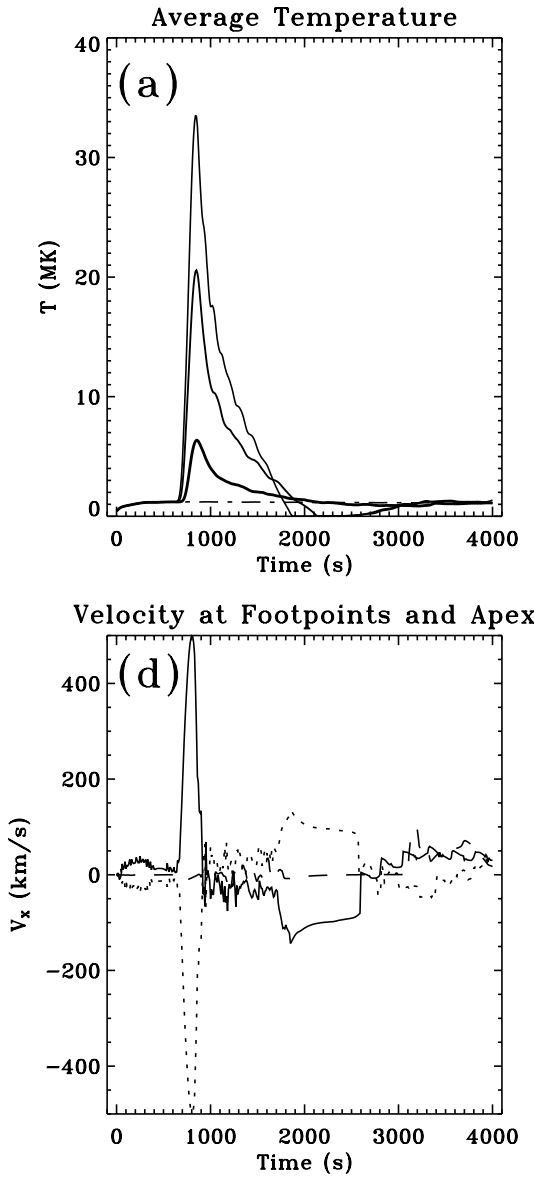
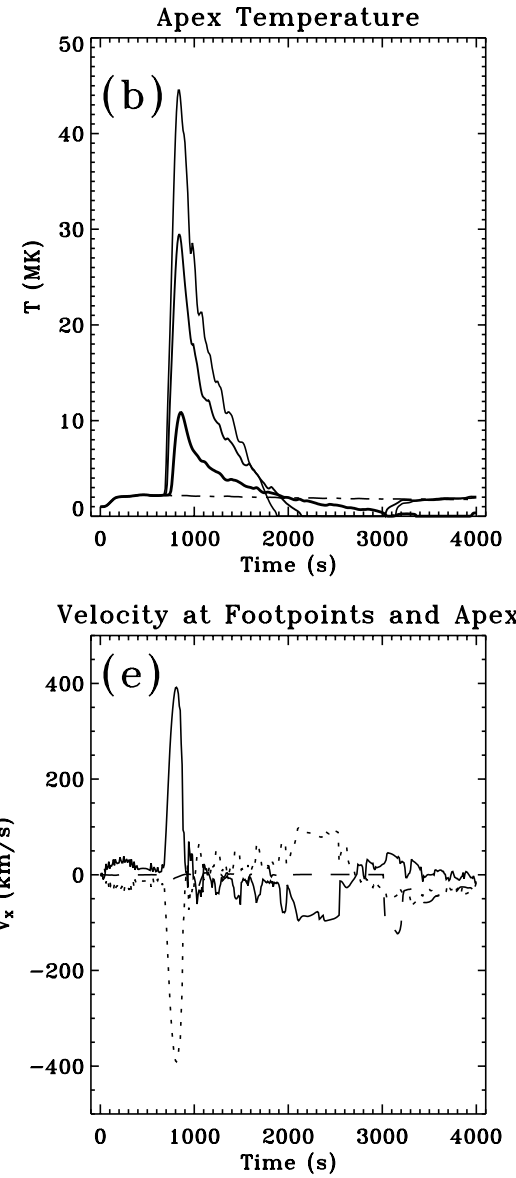
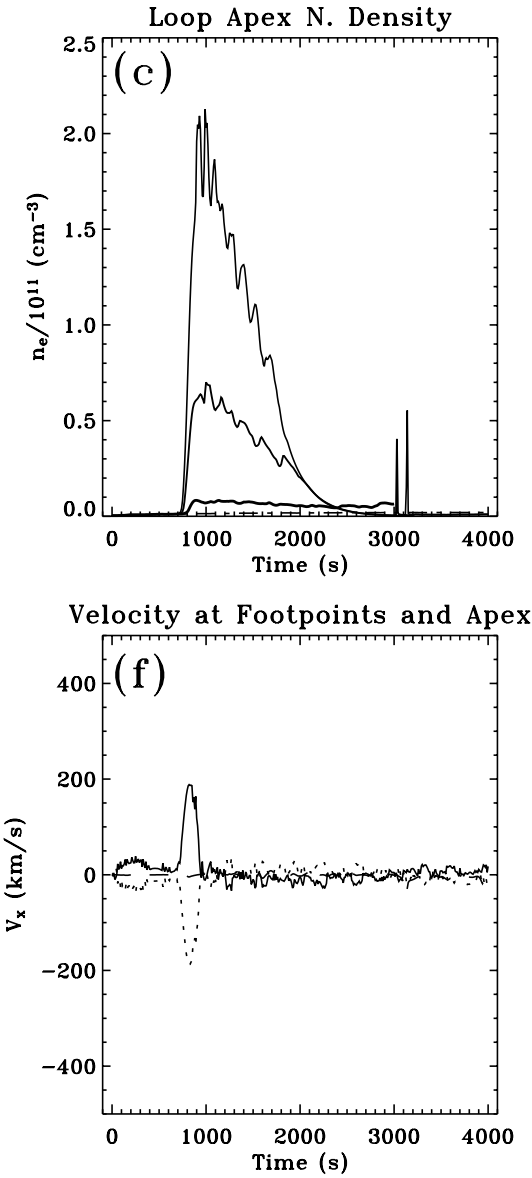

Fig. 4. The same as in Fig. 2, but for footpoint heating. However, the flare peak amplitudes $\alpha=0.01,0.25,1.0$ correspond here to the flare heating rates $\left(E_{\mathrm{H} 0}=1.5,37.5,150.0 \mathrm{erg} \mathrm{cm}^{-3} \mathrm{~s}^{-1}\right)$.

flaring loops. (2) In the case of footpoint heating, a flare takes less time on average (compare Figs. 3a and $3 \mathrm{~b}$ with Figs. 1a and $1 \mathrm{~b}$ ) - so the cooling of the loop happens faster. This can be explained by the following reason: at the initial stages of cooling conductive losses dominate over radiative losses (when $T \geq 20 \mathrm{MK}$ ), while the situation reverses (when $T \leq 20 \mathrm{MK}$ ) as the loop cools down and becomes less dense - plasma is flowing out of the loop (strong down flows were seen to accompany the cooling process in all of our numerical simulations and this is also confirmed in a number of observations). The time scale of conduction loss is proportional to the density, while the time scale of radiative loss is proportional to the inverse density (Aschwanden \& Alexander 2001). Therefore, since the radiative losses dominate over the heat conduction losses for most of the time, it is natural to conclude that the denser loops would cool faster. (3) In the case of footpoint heating the peak apex temperatures (corresponding to the same, as in the case of apex heating, average temperature of about $30 \mathrm{MK}$ ) are significantly lower (less by about $10 \mathrm{MK}$ ). The observational data of the Bastille-day flare, namely the temperature evolution (cf. top left panel of Fig. 11 in Aschwanden \& Alexander 2001), was derived for the entire field of view of the instrument, i.e. it tracks the dynamics of the average temperature. However, if one would have additional temperature dynamics at a loop given point such as at the apex, our simulations would allow to discriminate between the different heating functions of the loop during the flare. (4) In the case of footpoint heating up-flow velocities are somewhat higher (compare Figs. 3d-f with Figs. 1d-f).

To complete the comparison between apex and footpoint heating cases, we investigate the effect of the flare heating amplitude, by fixing the duration of the flare to $\sigma_{\mathrm{t}}=41 \mathrm{~s}$, and vary $\alpha=0.01,0.25,1.0$ (again with fixed $Q_{\mathrm{p}}=1.5 \times 10^{4}$ ). The results of these numerical runs are presented in Fig. 4 (see for comparison Fig. 2). Most of the features seen in the case of apex heating are still valid in this case. A notable difference is that, in the considered parameter space, spikes in the apex density time profiles occur (Fig. 4c), which can be also seen in Fig. 3c after the flare. In fact, these spikes are present in all runs with footpoint heating. They occur when dense blobs of plasma (prominences), formed by the thermal instability, swipe through the apex. One should mention, however, that a 1D code does not provide a fully adequate description of prominences, since it ignores the finite magnetic field tension (we use an infinite magnetic field limit in our code). In fact, this is the reason why they do not stay steady at the apex, as the magnetic field in our case cannot bend to provide cavity for a stationary prominence. Yet another difference between the two cases are the higher obtained densities and up-flow velocities during the flare in the case footpoint heating (due to more efficient 

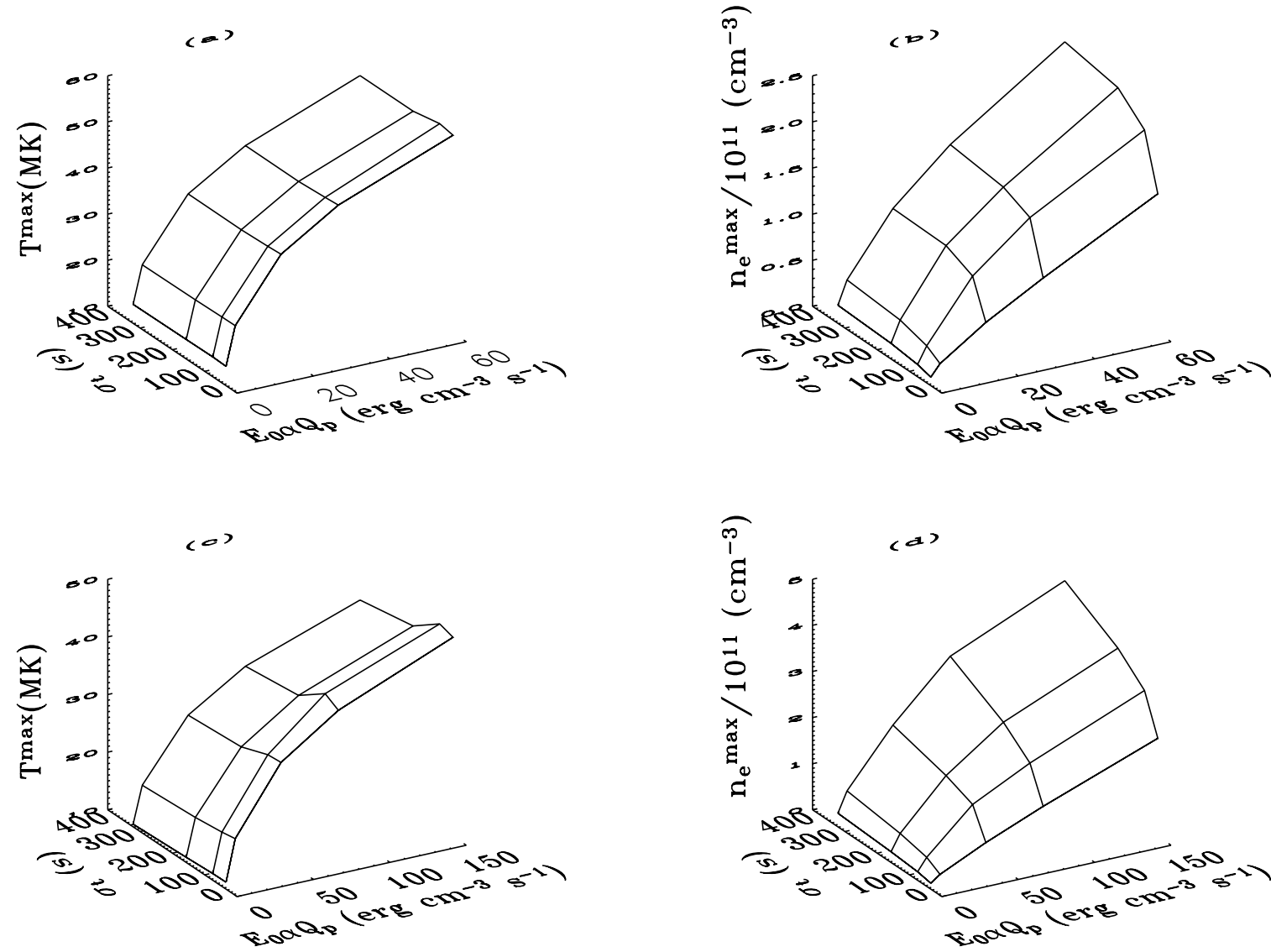

Fig. 5. a) The dependence of maximum obtained temperatures at the loop apex as a function of flare duration, $\sigma_{\mathrm{t}}$ and heating rate $E_{\mathrm{H} 0}$ for the case of apex heating. b) The same as in a), but for the maximum obtained density in the case of apex heating. c) The same as in a), but for the case of footpoint heating. d) The same as in b), but for footpoint heating.

evaporation) and lower obtained apex temperatures (due to the spatial flatness of the heating function along the loop).

\subsection{Parametric study}

In this subsection we present a parametric study of the problem by investigating the maximum obtained temperatures and densities at the loop apex as a function of flare duration, $\sigma_{\mathrm{t}}$ and the heating rate $E_{\mathrm{H} 0}=E_{0} \alpha Q_{\mathrm{p}}$. We have performed simulations for $\sigma_{\mathrm{t}}=41,82,164,329 \mathrm{~s}$ and $\alpha=$ $0.01,0.05,0.25,0.5,1.0$ with $Q_{\mathrm{p}}=3 \times 10^{4}$ for the case of apex heating and $Q_{\mathrm{p}}=1.5 \times 10^{4}$ in the case of footpoint heating. With appropriate multiplication by $E_{0}$ in each case $\left(E_{0}=0.002 \mathrm{erg} \mathrm{cm}^{-3} \mathrm{~s}^{-1}\right.$ for apex heating and $E_{0}=$ $0.01 \mathrm{erg} \mathrm{cm}^{-3} \mathrm{~s}^{-1}$ for footpoint heating), the flare heating rates, $E_{\mathrm{H} 0}$, are, $0.6,3.0,15.0,30.0,60.0 \mathrm{erg} \mathrm{cm}^{-3} \mathrm{~s}^{-1}$ in the case of apex heating and 1.5, 7.5, 37.5, 75.0,150.0 $\mathrm{erg} \mathrm{cm}^{-3} \mathrm{~s}^{-1}$ in the case of footpoint heating. The results of these numerical runs are presented in Fig. 5. The actual data that was used to produce Fig. 5 is given in Table 1. In Figs. 5a and 5c we plot the maximum obtained temperatures, $T^{\max }(\mathrm{MK})$, at the loop apex in the case of apex and footpoint heating, respectively. We gather from these plots that: (1) in both cases $T^{\max }$ is practically independent of the flare duration $\sigma_{\mathrm{t}}$ and it increases with the increase of the flare heating rate $E_{\mathrm{H} 0}$; (2) $T^{\max }$ is up to $10 \mathrm{MK}$ higher in the case of apex heating than in the case of footpoint heating. This is due to the steepness of the temperature profile along the loop in the case of apex heating (corresponding to the same average temperature as in the case of footpoint heating). In Figs. 5b and 5d we plot the maximum obtained densities, $n_{\mathrm{e}}^{\max } / 10^{11}\left(\mathrm{~cm}^{-3}\right)$, at the loop apex in the case of apex and footpoint heating, respectively. It can be seen that (1) In both cases $n_{\mathrm{e}}^{\max }$ increases with the increase of both the flare heating rate $E_{\mathrm{H} 0}$ and flare duration $\sigma_{\mathrm{t}}$, and (2) $n_{\mathrm{e}}^{\max }$ is about twice as high in the case of footpoint heating than in the case of apex heating. This is due to more efficient material evaporation from the chromosphere and the region connecting corona to the chromosphere in the case of footpoint heating. We have also performed parametric study varying the loop length. The results are presented in Table 2 . Note that for consistent comparison with other numerical runs, in the case of footpoint heating, when varying loop length, we have shifted $s_{0}$ so that spatial maximum of the heating function always stayed at the top of chromosphere. We have varied the loop length in the range of $L=(0.25, \ldots, 2.0) \times L_{0}$ (with $L_{0}=55 \mathrm{Mm}$, cf. Table 2). We find that the loop average temperature does not change dramatically, while the loop apex temperature increases notably for longer loops only for the case of apex heating, but much less for footpoint heating. Also we find that the mean electron density decreases somewhat for longer loops, i.e., $n_{\mathrm{e}} / 10^{11}\left(\mathrm{~cm}^{-3}\right)=10.45 L^{-0.362}$ for apex heating and $n_{\mathrm{e}} / 10^{11}\left(\mathrm{~cm}^{-3}\right)=9.62 L^{-0.207}$ for footpoint 
Table 1. Flare loop temperatures and densities as function of the heating rate and heating duration, for a fixed loop length of $L=55 \mathrm{Mm}$, obtained from 1D hydrodynamic simulations. Units of $E_{\mathrm{H} 0}$ are in (erg $\mathrm{cm}^{-3} \mathrm{~s}^{-1}$ ). $\sigma_{\mathrm{t}}$ is measured in seconds, while temperatures and densities are given in (MK) and $\left(\mathrm{cm}^{-3}\right)$ respectively.

\begin{tabular}{|c|c|c|c|c|c|}
\hline $\begin{array}{l}\text { Heat. } \\
\text { locat. } \\
\text { Ap./Ft. }\end{array}$ & $\begin{array}{l}\text { Heat. } \\
\text { rate } \\
E_{\mathrm{H} 0}\end{array}$ & $\begin{array}{l}\text { Heat. } \\
\text { durat. } \\
\sigma_{\mathrm{t}}\end{array}$ & $\begin{array}{l}\text { Temp. } \\
\text { at ap. } \\
T^{\max }\end{array}$ & $\begin{array}{l}\text { Temp. } \\
\text { aver. } \\
T_{\text {avg }}\end{array}$ & $\begin{array}{l}\text { Elect. } \\
\text { dens. } \\
n_{\mathrm{e}}^{\max } / 10^{11}\end{array}$ \\
\hline $\mathrm{A}$ & 0.60 & 41 & 13.75 & 6.78 & 0.07 \\
\hline A & 0.60 & 82 & 13.86 & 7.26 & 0.11 \\
\hline A & 0.60 & 164 & 13.78 & 7.44 & 0.15 \\
\hline A & 0.60 & 329 & 13.67 & 7.57 & 0.17 \\
\hline $\mathrm{F}$ & 1.50 & 41 & 10.84 & 6.35 & 0.08 \\
\hline $\mathrm{F}$ & 1.50 & 82 & 10.80 & 6.93 & 0.14 \\
\hline $\mathrm{F}$ & 1.50 & 164 & 10.53 & 7.06 & 0.19 \\
\hline F & 1.50 & 329 & 10.11 & 7.10 & 0.25 \\
\hline A & 3.00 & 41 & 22.12 & 11.80 & 0.19 \\
\hline A & 3.00 & 82 & 22.07 & 12.46 & 0.29 \\
\hline A & 3.00 & 164 & 21.94 & 12.72 & 0.38 \\
\hline A & 3.00 & 329 & 21.80 & 12.94 & 0.43 \\
\hline F & 7.50 & 41 & 18.04 & 11.52 & 0.23 \\
\hline $\mathrm{F}$ & 7.50 & 82 & 17.64 & 12.22 & 0.41 \\
\hline $\mathrm{F}$ & 7.50 & 164 & 17.09 & 12.37 & 0.54 \\
\hline F & 7.50 & 329 & 16.51 & 12.42 & 0.69 \\
\hline A & 15.00 & 41 & 35.38 & 20.38 & 0.53 \\
\hline A & 15.00 & 82 & 35.18 & 21.34 & 0.94 \\
\hline A & 15.00 & 164 & 34.90 & 21.53 & 1.07 \\
\hline A & 15.00 & 329 & 34.95 & 21.78 & 1.09 \\
\hline $\mathrm{F}$ & 37.50 & 41 & 29.50 & 20.57 & 0.70 \\
\hline $\mathrm{F}$ & 37.50 & 82 & 29.28 & 21.48 & 1.33 \\
\hline F & 37.50 & 164 & 27.56 & 21.36 & 1.57 \\
\hline F & 37.50 & 329 & 26.88 & 21.31 & 1.90 \\
\hline A & 30.00 & 41 & 43.26 & 25.72 & 0.87 \\
\hline A & 30.00 & 82 & 42.99 & 26.80 & 1.43 \\
\hline A & 30.00 & 164 & 42.59 & 26.87 & 1.57 \\
\hline A & 30.00 & 329 & 42.64 & 27.10 & 1.64 \\
\hline F & 75.00 & 41 & 36.30 & 26.29 & 1.21 \\
\hline $\mathrm{F}$ & 75.00 & 82 & 37.65 & 26.73 & 1.95 \\
\hline F & 75.00 & 164 & 34.26 & 26.74 & 2.45 \\
\hline $\mathrm{F}$ & 75.00 & 329 & 33.15 & 26.46 & 3.11 \\
\hline A & 60.00 & 41 & 52.85 & 32.42 & 1.51 \\
\hline A & 60.00 & 82 & 53.44 & 33.05 & 2.10 \\
\hline A & 60.00 & 164 & 52.27 & 33.29 & 2.37 \\
\hline A & 60.00 & 329 & 52.27 & 33.49 & 2.48 \\
\hline F & 150.00 & 41 & 44.54 & 33.52 & 2.13 \\
\hline F & 150.00 & 82 & 45.31 & 34.18 & 2.97 \\
\hline $\mathrm{F}$ & 150.00 & 164 & 41.87 & 33.05 & 3.50 \\
\hline F & 150.00 & 329 & 40.19 & 32.86 & 4.19 \\
\hline
\end{tabular}

heating. Here, $L$ is loop length in $\mathrm{Mm}$. The key point is that in longer loops thermal conduction is less efficient in working its way through the loop and evaporating chromospheric plasma,
Table 2. Flare loop temperatures and densities as function of the loop length (in Mm), for a fixed heating duration of $\sigma_{\mathrm{t}}=329 \mathrm{~s}$. Units of $E_{\mathrm{H} 0}$ are in $\left(\mathrm{erg} \mathrm{cm}^{-3} \mathrm{~s}^{-1}\right) . \sigma_{\mathrm{t}}$ is measured in seconds, while temperatures and densities are given in (MK) and $\left(\mathrm{cm}^{-3}\right)$ respectively.

\begin{tabular}{|c|c|c|c|c|c|}
\hline $\begin{array}{l}\text { Heat. } \\
\text { locat. } \\
\text { Ap./Ft. }\end{array}$ & $\begin{array}{l}\text { Heat. } \\
\text { rate } \\
E_{\mathrm{H} 0}\end{array}$ & $\begin{array}{l}\text { Temp. } \\
\text { at ap. } \\
T^{\max }\end{array}$ & $\begin{array}{l}\text { Temp. } \\
\text { aver. } \\
T_{\text {avg }}\end{array}$ & $\begin{array}{l}\text { Elect. } \\
\text { dens. } \\
n_{\mathrm{e}}^{\max } / 10^{11}\end{array}$ & $\begin{array}{l}\text { Loop } \\
\text { leng. } \\
L[\mathrm{Mm}]\end{array}$ \\
\hline A & 60.00 & 35.19 & 28.18 & 3.77 & 13.75 \\
\hline A & 60.00 & 42.12 & 31.67 & 3.30 & 27.50 \\
\hline A & 60.00 & 47.72 & 32.88 & 2.89 & 41.25 \\
\hline A & 60.00 & 52.27 & 33.49 & 2.48 & 55.00 \\
\hline A & 60.00 & 55.46 & 33.94 & 2.30 & 68.75 \\
\hline A & 60.00 & 58.77 & 34.47 & 2.17 & 82.50 \\
\hline A & 60.00 & 61.23 & 34.74 & 1.88 & 96.25 \\
\hline A & 60.00 & 63.57 & 34.98 & 1.83 & 110.00 \\
\hline $\mathrm{F}$ & 150.00 & 38.18 & 31.84 & 5.54 & 13.75 \\
\hline F & 150.00 & 39.33 & 32.58 & 4.61 & 27.50 \\
\hline $\mathrm{F}$ & 150.00 & 40.35 & 32.89 & 4.89 & 41.25 \\
\hline $\mathrm{F}$ & 150.00 & 40.19 & 32.86 & 4.19 & 55.00 \\
\hline F & 150.00 & 40.64 & 32.85 & 4.02 & 68.75 \\
\hline $\mathrm{F}$ & 150.00 & 40.86 & 32.92 & 3.85 & 82.50 \\
\hline F & 150.00 & 42.61 & 33.24 & 3.70 & 96.25 \\
\hline F & 150.00 & 43.13 & 33.23 & 3.56 & 110.00 \\
\hline
\end{tabular}

since the characteristic time scale of the thermal conduction is proportional to $L^{2}$. This is the reason for having the less dense loops when their length is increased. In turn, less dense loops cool less efficiently, and thus one ends up with increased loop temperatures for the longer loops.

\section{Conclusions}

In summary, we have used a radiative hydrodynamic numerical code to simulate flares. The physical parameters of the input were obtained from observations of the the Bastille-day flare (Aschwanden \& Alexander 2001). Our simulations confirm the general picture of flare dynamics: transient heat deposition either at loop footpoints (with maximum heat input at the bottom of the region connecting corona to the chromosphere or i.e. top of chromosphere) or at the apex leads to an average loop temperature of $T_{\mathrm{avg}} \approx 30 \mathrm{MK}$ first. Then, evaporation of material from the chromosphere and the region connecting corona to the chromosphere into corona ensues with up-flows in the order of a few hundreds of $\mathrm{km} \mathrm{s}^{-1}$. During the peak of the flare, the combined action of heat input and conductive and radiate loss yields an oscillatory flow pattern with typical amplitudes of up to few tens of $\mathrm{km} \mathrm{s}^{-1}$. Finally we enter a cooling phase, when down-flows in the order of hundred $\mathrm{km} \mathrm{s}^{-1}$ can be seen as the plasma drains out of the loop, ultimately reaching an equilibrium.

We have established the following:

1. In the case of footpoint heating, the obtained maximum values of the density are considerably higher $\left(4.2 \times 10^{11} \mathrm{~cm}^{-3}\right.$ or more) than in the case of apex heating $\left(2.5 \times 10^{11} \mathrm{~cm}^{-3}\right)$. 
This is due to the fact that footpoint heating is more efficient in evaporating material from the region connecting corona to the chromosphere and chromosphere itself, which yields denser loops during the flare. In the case of apex heating, which was used to model flares, insufficient downward heating conduction prevents significant material evaporation.

2. In the case of footpoint heating, as compared to the apex heating, on average cooling after the flare takes less time. This due to the fact that the time scale of conduction loss is proportional to the density, while the time scale of radiative loss is reciprocal to the density (Aschwanden \& Alexander 2001). Therefore, since radiative losses dominate over heat conduction losses for most of the time, it is clear that the denser loops cool faster.

3. In principle, our simulations would allow to discriminate between different heating functions of the loop during the flare, if one would have temperature dynamics in a given point of the loop, such as at the apex. This is based on our observation that in the case of footpoint heating the peak apex temperatures (corresponding to the same, as in the case of apex heating, average temperature of about $30 \mathrm{MK}$ ) are significantly lower (less by about $10 \mathrm{MK}$ ).

4. In the case of footpoint heating, up-flow velocities are higher (roughly by up to $100 \mathrm{~km} \mathrm{~s}^{-1}$ ) than in the case of apex heating due to more efficient evaporation.

5. In both cases (of apex and footpoint heating) the maximum obtained temperature $T^{\max }$ at the loop apex is practically independent of the heating duration $\sigma_{\mathrm{t}}$, but it increases with higher heating rates $E_{\mathrm{H} 0}$.

6. The maximum obtained densities at the loop apex increase with the increase of both the flare heating rate $E_{\mathrm{H} 0}$ and the heating duration $\sigma_{\mathrm{t}}$, in the case of apex as well as footpoint heating.

7. Varying the loop length (see Table 2) in the range of $L=$ $(0.25, \ldots, 2.0) \times L_{0}$ (with $\left.L_{0}=55 \mathrm{Mm}\right)$, we find (1) that the mean loop temperature averaged over the loop length does not change dramatically; (2) that the loop apex temperature increases notably for longer loops only for the case of apex heating, but much less for footpoint heating, and (3) that the mean electron density decreases somewhat for longer loops, i.e., $n_{\mathrm{e}} / 10^{11}\left(\mathrm{~cm}^{-3}\right)=10.45 L^{-0.362}$ for apex heating and $n_{\mathrm{e}} / 10^{11}\left(\mathrm{~cm}^{-3}\right)=9.62 L^{-0.207}$ for footpoint heating. Here, $L$ is loop length in $\mathrm{Mm}$.

Also, in practically all of our numerical runs we have detected quasi-periodic oscillations in all physical quantities. In fact, such oscillations are frequently seen during solar flares (e.g. Terekhov et al. 2002) as well as stellar flares (e.g. Mathioudakis et al. 2003). Our preliminary analysis shows (Nakariakov et al. 2004; Tsiklauri et al. 2004) that quasi-periodic oscillations seen in our numerical simulations bear many similar features as the observed ones. The key point is that the traditional explanation of these oscillations in the observations involves $M H D$ waves. In the numerical simulations presented here, however, they are likely to be produced by standing sound waves caused by impulsive and localized heating. Therefore, our explanation of these oscillations is purely hydrodynamic - they are related to the standing slow mode acoustic waves, similar to the observed by SUMER (Wang et al. 2002). A detailed study of these quasi-periodic oscillations will be presented elsewhere (Nakariakov et al. 2004; Tsiklauri et al. 2004).

In a next step we plan to use the outputs of this parametric study of hydrodynamic simulations, which cover a wide parameter range of heating rates and heating time scales, as input for forward-fitting to the observed physical parameters (densities and temperatures) of the multi-loop flare on Bastille-day 2000 July 14.

Acknowledgements. This work was supported, in part, by PPARC, UK. Numerical calculations were performed using the PPARC funded MHD Cluster at St. Andrews. The work of M.J.A. was partially supported by NASA contract NAS5-38099 (TRACE). D.T. would like to thank the anonymous referee for very useful comments and suggestions on improving the manuscript.

\section{References}

Arber, T. D., Longbottom, A. W., Gerrard, C. L., \& Milne, A. M. 2001, J. Comput. Phys., 171, 151

Aschwanden, M. J., \& Alexander, D. 2001, Sol. Phys., 204, 93

Aschwanden, M. J., Brown, J. C., \& Kontar, E. P. 2002, Sol. Phys., 210,383

Aschwanden, M. J., Schrijver, C. J., \& Alexander, D. 2001, ApJ, 550, 1036

Braginskii, S. I. 1965, Transport processes in plasma, Reviews of Plasma Phys. (New York: Consultants Bureau), 1, 205

Fisher, G. H., \& Hawley, S. L. 1990, ApJ, 357, 243

Hori, K., Yokoyama, T., Kosugi, T., \& Shibata, K. 1997, ApJ, 489, 426

Hori, K., Yokoyama, T., Kosugi, T., \& Shibata, K. 1998, ApJ, 500, 492

Kopp, R. A., \& Pneuman, G. W. 1976, Sol. Phys., 50, 85

Leboef, J. N., Tajima, T., \& Dawson, J. M. 1982, Phys. Fluids, 25, 784

Mariska, J. T. 1987, ApJ, 319, 465

Mariska, J. T., Emslie, A. G., \& Li, P. 1989, ApJ, 341, 1067

Mathioudakis, M., Seiradakis, J. H., Williams, D. R., et al. 2003, A\&A, 403, 1101

Nakariakov, V. M., Tsiklauri, D., Kelly, A., Arber, T. D., \& Aschwanden, M. J. 2004, A\&A, 414, L25

Peres, G., Rosner, R., Serio, S., \& Vaiana, G. S. 1982, ApJ, 252, 791

Priest, E. R. 1982, Solar Magnetohydrodynamics (Dordrecht, Holland: D. Reidel Publ. Comp.)

Priest, E. R., \& Forbes, T. 2000, Magnetic Reconnection (Cambridge University Press)

Rosner, R., Tucker, W. H., \& Vaiana, G. S. 1978, ApJ, 220, 643

Sturrock, P. A. 1966, Nature, 5050, 695

Terekhov, O. V., Shevchenko, A. V., Kuz'min, A. G., et al. 2002, Astron. Lett., 28, 397

Tsiklauri, D., \& Nakariakov, V. M. 2001, A\&A, 379, 1106

Tsiklauri, D., Nakariakov, V. M., Arber, T. D., \& Aschwanden, M. J. 2004, A\&A, accepted

Wang, T., Solanki, S. K., Curdt, W., Innes, D. E., \& Dammasch, I. E. 2002, ApJ, 574, L101

Warren, H. P., Wineberger, A. R., \& Mariska, J. T. 2003, ApJ, 593, 1174 